\title{
Schisandrin B Enhances Glutathione Redox Cycling and Protects Against $\beta$-amyloid-induced Apoptosis in Human Neuroblastoma SH-SY5Y Cells: A Comparative Study of Various Phytochemicals
}

\author{
P. K. Leong, P. Y. Lam, N. Chen and K. M. Ko*
}

Division of Life Science, The Hong Kong University of Science \& Technology, Clear Water Bay, Hong Kong SAR, China

\begin{abstract}
In the present study, we aim to define the cytoprotective mechanism of (-)schisandrin B [(-)Sch B] in comparison with other phytochemicals in SH-SY5Y cells. The effects of (-)Sch B and curcumin (Cur), resveratrol (Rev) and epigallocatechin gallate (EGCG) on $\beta$-amyloid (A $\beta$ )-induced apoptosis were investigated in SH-SY5Y cells. Cellular reduced glutathione (GSH) levels and peroxide-induced GSH depletion were measured. Activities of glutathione reductase (GR) and glucose-6-phosphate dehydrogenase $(\mathrm{G} 6 \mathrm{DPH})$ in $\mathrm{A} \beta$-challenged cells were also examined. All tested phytochemicals were investigated for the activation of nuclear factor erythroid-2 related factor 2 (Nrf2) in SH-SY5Y cells, using a luciferase-based assay. Finally, they were examined for the effect on the extent of phosphorylation of Tau in A $\beta$ challenged cells. The results showed that only (-)Sch B and EGCG protected against A $\beta$-induced apoptosis in SH-SY5Y cells. The cytoprotection afforded by (-)Sch B and EGCG were associated with an increase in cellular GSH levels in A $\beta$ challenged cells and a reduction in peroxide-induced GSH depletion. However, only (-)Sch B, but not EGCG, increased G6DPH and GR activities in A $\beta$-challenged cells and caused the activation of Nrf2 in unchallenged cells. Both (-)Sch B and EGCG reduced the extent of Tau phosphorylayion in A $\beta$-challenged cells. In conclusion, (-)Sch B may enhance cellular glutathione redox cycling, presumably by increasing G6PDH and GR activities, via activation of the Nrf2 signaling pathway, whereas EGCG likely acts as a radical scavenger. Both (-)Sch B and EGCG suppressed the phosphorylation of Tau in $\mathrm{A} \beta$-challenged cells, suggesting their potential in ameliorating the pathological condition of Alzheimer's disease.
\end{abstract}

Keywords: Antioxidant, beta-amyloid, glutathione, Nrf2, phytochemicals, schisandrin B.

\section{INTRODUCTION}

Alzheimer's disease is the most common form of agerelated progressive neurologic disorder that results in memory loss, cognitive impairment and even death [1]. While Alzheimer's disease is reaching epidemic proportions in an increasing by aged population world-wide, effective therapeutic interventions are currently unavailable for its treatment [2]. Although the pathological mechanism(s) underlying the development of Alzheimer's disease remain(s) relatively unclear, a large body of evidence has suggested the involvement of oxidative stress in its pathogenesis [3]. In this connection, a wide array of phytochemicals (such as polyphenolics isolated from plants) that possess antioxidant properties have been investigated as potential candidates for preventing or at least retarding the progression of Alzheimer's disease [4]. Schisandrin B (Sch $\mathrm{B}$ ), which is the most abundant active dibenzocyclooctadiene lignan (i.e. a class of non-flavonoid polyphenolics) in the fruit of Schisandra chinensis, was found to produce nonspecific protection against tissue oxidant injury in various

*Address correspondence to the author at the Division of Life Science, The Hong Kong University of Science \& Technology, Clear Water Bay, Hong Kong SAR, China; Tel: +852-23587298; Fax: +852-27198158;

E-mail: bcrko@ust.hk organs of rodents, presumably by up-regulating the glutathione redox cycling [5-7]. Previous studies have also demonstrated that $(-)$ Sch B, a potent stereoisomer of Sch B that amounts to $80 \%$ of the stereoisomers [8], increases the resistance of neuronal cells to paraquat- and 3-nitroponoic acid-induced oxidative stress through reducing the extent of oxidant-induced reduced glutathione (GSH) depletion, indicative of enhanced glutathione redox cycling $[9,10]$. It is therefore of pharmacological interest to examine whether $(-)$ Sch B can counteract oxidative stress-induced neuronal cell apoptosis, which has been implicated in the development of Alzheimer's disease. To further explore the role of antioxidant status in determining the cytoprotective action of $(-)$ Sch B, its effects were compared to those of three phytochemicals isolated from other herbs/plants as subclasses of polyphenols, namely, curcumin (Cur, a nonflavonoid polyphenol), resveratrol (Rev, a stilbene) and epigallocatechin gallate (EGCG, a flavanol), all of which have been shown to possess antioxidant properties [11].

It has been suggested that the long-term increase in oxidative stress caused by the progressive accumulation of aggregated $A \beta$ peptides in the brain of Alzheimer's disease patients during the course of the disease may result in neurodegeneration [12]. In this regard, $A \beta_{25-35}$, which contains a functional domain of the full length $A \beta$ and is 
required for both neurotrophic and neurotoxic effects, has been widely used as a pharmacological tool for establishing experimental models of Alzheimer's disease [13, 14]. In the present study, an in vitro human neuroblastoma SH-SY5Y cell model was utilized to investigate the effects of (-)Sch B and three other phytochemicals on $\mathrm{A} \beta_{25-35}$-induced apoptotic cell death. (-)Sch B and other phytochemical-induced changes in cellular GSH levels and in the activities of glucose-6-phosphate dehydrogenase (G6PDH) and glutathione reductase (GR), two enzymes critically involved in glutathione redox cycling, were investigated in SH-SY5Y cells under both control and $A \beta$-challenged conditions. To investigate the involvement of enhancement in glutathione redox cycling, the effects of $(-)$ Sch $B$ and the other phytochemicals on tert-butyhydroperoxide ( $t$-BHP)-induced GSH depletion were studied. $t$-BHP, but not hydrogen peroxide, was found to produce an oxidative stress in a consistent manner by depleting cellular GSH, as well as inhibiting related antioxidant components in HepG2 hepatocytes [15]. The ability of $t$-BHP to deplete cellular GSH levels was also observed in cultured cardiomyocytes, hepatocytes and neuronal cells [16]. (-)Sch B and other phytochemicals were examined for their ability to activate nuclear factor erythroid-2 related factor 2 (Nrf2), a redoxsensitive transcription activator of antioxidant genes using a luciferase-based assay. Finally, their effects on A $\beta$-induced phosphorylation of Tau protein, a pathological hallmark of Alzheimer's disease, were assessed by Western blot analysis.

\section{MATERIALS AND METHODS}

\section{Chemicals and Cell Culture Materials}

All chemicals were purchased from Sigma Chemical Co. (St Louis, MO, USA) unless otherwise specified. Dulbecco's modified eagle cell culture medium (DMEM), fetal bovine serum and Lipofectamine 2000 were purchased from Invitrogen (Grand Island, NY, USA). The fruit of Schisandra chinensis was imported from China, and it was authenticated and supplied by a commercial dealer Lee Hoong Kee Ltd. (Hong Kong SAR, China). (-)Sch B was purified from a petroleum ether extract of the fruit of Schisandra chinensis by silica gel chromatography, followed by preparative C18 HPLC, as previously described [17]. The purity of $(-)$ Sch B was $>90 \%$, as determined by HPLC analysis [17]. All other chemicals were of analytical grade.

\section{Cell Culture, Phytochemical Pre-incubation and A $\beta$ challenge}

A human neuroblastoma clonal SH-SY5Y cell line was purchased from American Type Culture Collection (Manassas, VA, USA) and maintained in DMEM supplemented with $10 \%$ fetal bovine serum. Cells used for experimentation were seeded at a density of $5 \times 10^{5}$ cells/well on a 6-well cell culture dish or $2 \times 10^{5}$ cells/well on a 24-well cell culture dish when transfection with Nrf2luciferase plasmid was performed. $A \beta$ was purchased from Sigma Chemical Co (St Louis, MO). and dissolved in sterile deionized water, and stored at $-20{ }^{\circ} \mathrm{C}$ prior to use. To obtain the neurotoxic form of $A \beta$, solution of the peptide was placed in an incubator at $37^{\circ} \mathrm{C}$ for 7 days prior to performing the experiments [5]. Cells were incubated with various concentrations of phytochemical [5 or $15 \mu \mathrm{M}$ for (-)Sch B, Res or EGCG; 1 or $2.5 \mu \mathrm{M}$ for Cur] for $6 \mathrm{~h}$. The phytochemical-containing medium was then replaced with normal medium, and the cultured cells were incubated for an additional $16 \mathrm{~h}$ prior to $\mathrm{A} \beta(40 \mu \mathrm{M})$ challenge which involved an incubation for $48 \mathrm{~h}$. According to a preliminary study, the 16-h incubation post-(-)Sch B exposure results in a maximal increase in cellular GSH levels in SH-SY5Y cells. Cell lysates were then prepared and subjected to biochemical analyses.

\section{Preparation of Cell Lysates}

SH-SY5Y cells were harvested by trypsinization and the cells were then resuspended in lysis buffer $[20 \mathrm{mM}$ TrisHCl, $2 \mathrm{mM}$ ethylenediaminetetraacetic acid (EDTA), $3 \mathrm{mM}$ ethylene glycol tetraacetic acid (EGTA), $1 \%$ (v/v) Triton X$100,10 \%$ (v/v) glycerol, $2 \mathrm{mM}$ dithiothreitol, $\mathrm{pH}$ 7.5] and incubated on ice for $5 \mathrm{~min}$. The cells were then centrifuged at $14,000 \times g$ for $10 \mathrm{~min}$ at $4{ }^{\circ} \mathrm{C}$, and the resultant supernatant was subjected to biochemical and Western blot analysis.

\section{Biochemical Analysis}

A $\beta$-induced apoptotic cell death was assessed by the fluorometric measurement of caspase activation using a commercially available assay kit (PerkinElmer, Boston, MA, USA). GSH levels in cell lysates were measured using the enzymatic method described by Griffith [18]. The degree of GSH depletion induced by $t$-BHP challenge was estimated by comparing the GSH level at $1 \mathrm{~min}$ post- $t$-BHP challenge with the basal value (i.e., at $0 \mathrm{~min}$ ) and expressed as \% depletion. GR and G6DPH activities were measured as described by Seelig and Meister [19] and Ip et al., [20] respectively.

\section{Nrf2-luciferase Reporter Assay}

Nrf2-luciferase reporter, which consists of a Nrf2responsive firefly luciferase construct and a constitutively expressing Renilla luciferase construct at a ratio of 40:1, was purchased from SABiosciences (Frederick, MD, USA). In brief, SH-SY5Y cells were grown to $70-80 \%$ confluence in a 24-well cell culture dish and transfected with Nrf2-luciferase reporter $(0.5 \mu \mathrm{g} /$ well $)$ using Lipofectamine 2000 according to the manufacturer's protocol. Twenty-four hours later, the transfection mixture was replaced by fresh medium, and the transfected cells were incubated with different concentrations of phytochemicals $(15 \mu \mathrm{M}$ for $(-)$ Sch B, Res or EGCG; $2.5 \mu \mathrm{M}$ for Cur) or vehicle for $6 \mathrm{~h}$. The phytochemical- or vehicle-containing medium was replaced by normal medium, and the cultured cells were further incubated for $16 \mathrm{~h}$. The phytochemical-pre-incubated cells were washed with phosphate-buffered saline and then with the lysis buffer provided by the dual-luciferase reporter assay kit [Promega (Madison, WI, USA)]. Luciferase activities in the cell lysates were then measured. The luminescence of samples was measured using a Victor 3 Multi-label Counter (Perkin Elmer, Turku, Finland). Renilla luciferase expression 
was used to normalize the varying transfection in the samples.

\section{Western Blot Analysis}

SH-SY5Y cell lysate $(50 \mu \mathrm{g})$ was mixed with Laemmli's loading buffer, boiled for $5 \mathrm{~min}$, and separated by electrophoresis (SDS-PAGE) on a $10 \%$ sodium dodecyl sulfate-polyacrylamide gel at $140 \mathrm{~V}$ followed by electroblotting to nitrocellulose membranes [purchased from Bio-Rad, (Hercules, CA, USA)] for $2 \mathrm{~h}$ at $100 \mathrm{~V}$. Membranes were blocked for $1 \mathrm{~h}$ with $5 \%$ non-fat milk in Tris-buffered saline with Tween-20 (TBS-T, $50 \mathrm{mM}$ Tris, $150 \mathrm{mM} \mathrm{NaCl}, 0.05 \%$ Tween-20) at room temperature and subsequently probed overnight with anti-phosphorylated Tau [1:2000, purchased from Santa Cruz Biotechnology Inc. (Santa Cruz CA, USA)] or anti-Tau [1:2000, purchased from BD Pharmingen (San Diego, CA, USA)]. The membranes were rinsed with TBS-T and incubated with a horseradish peroxidase-conjugated secondary antibody (1:5000, purchased from Santa Cruz). Following the incubation, the membranes were rinsed with TBS-T, and bound antibodies were detected by using enhanced chemiluminescence following the manufacturer's instructions (Cell Signaling Technology Inc., Danvers, MA, USA). Individual membranes were stripped and probed for anti- $\beta$-actin
(1:5000, Sigma) used as loading control. Individual band densities were quantified by ImageJ software (NIH, USA).

\section{Protein Assay}

Protein concentrations of cell lysates were determined using a Bio-Rad protein assay kit (catalog number: 5000006), which is a colorimetric assay using the Bradford dyebinding method. The absorbance of samples was then measured at $600 \mathrm{~nm}$. Bovine serum albumin was used as standard.

\section{Statistical Analysis}

Data were analyzed by one-way analysis of variance and intergroup differences were detected by the Scheffe method (single-step multiple comparison), with a value of $p<0.05$. Data from at least three independent experiments were expressed as means \pm S.D.

\section{RESULTS}

\section{$(-)$ Sch $B$ and EGCG protect against A $\beta$-induced apoptosis}

A $\beta$ challenge caused apoptotic cell death in SH-SY5Y cells, as evidenced by a significant increase ( 2.4 fold) in the activity of caspase-3, when compared with the unchallenged
A)

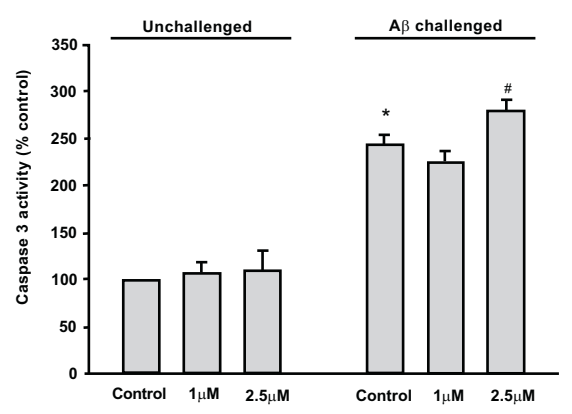

C)

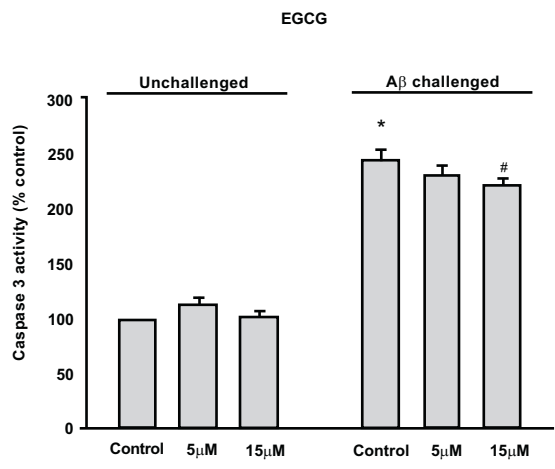

B)
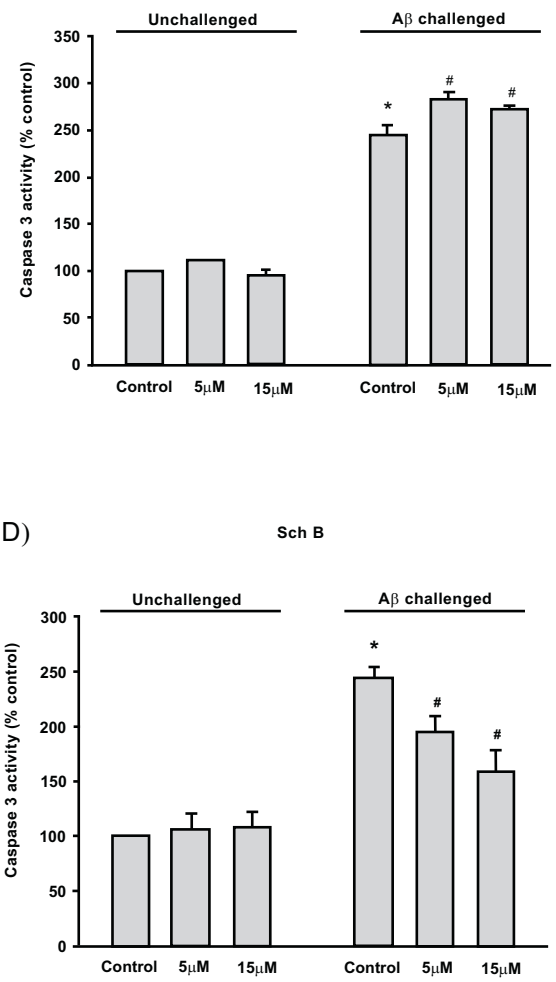

Fig. (1). Effects of (-)Sch B, curcumin, resveratrol and EGCG on A $\beta$-induced apoptosis in SH-SY5Y cells. Cells were pre-incubated with different concentrations of phytochemical ( 5 or $15 \mu \mathrm{M}$ for $(-)$ Sch B, resveratrol or EGCG; 1 or $2.5 \mu \mathrm{M}$ for curcumin) prior to A $\beta$ challenge. The extent of apoptosis was assessed by caspase-3 activation as described in methods and materials. Values given are means \pm SD, with $\mathrm{n} \geq$ 3. * Significantly different from the unchallenged control group $(P<0.05)$; \# significantly different from the A $\beta-$ challenged control group $(P<0.05)$. 
control. Pre-incubation with (-)Sch B (5 or $15 \mu \mathrm{M})$ dosedependently reduced the $A \beta$-induced increase in the extent of apoptosis (by 20 and 35\%, respectively) (Fig. 1A). EGCG $(15 \mu \mathrm{M})$ also significantly reduced $A \beta$-induced apoptotic cell death by $9.3 \%$ (Fig. 1D). While Cur $(1 \mu \mathrm{M})$ did not affect the degree of apoptosis in A $\beta$ - challenged SH-SY5Y cells, Cur $(2.5 \mu \mathrm{M})$ and $\operatorname{Rev}(5 \mu \mathrm{M})$ significantly exacerbated the degree of apoptotic cell death (by 15\% and $16 \%$, respectively), when compared with the $\mathrm{A} \beta$-challenged controls (Fig. 1B, 1C).

\section{$(-)$ Sch $B$ and EGCG suppress the A $\beta$ - and t-BHP- induced cellular GSH depletion}

Whereas pre-incubation with phytochemicals did not alter cellular GSH levels in unchallenged SH-SY5Y cells, $\mathrm{A} \beta$ challenge caused a significant reduction in cellular GSH content (by 38\%), when compared with the unchallenged controls (Fig. 2). (-)Sch B $(15 \mu \mathrm{M})$ and EGCG $(15 \mu \mathrm{M})$ significantly suppressed the $A \beta$-induced depletion of cellular GSH (by 13 and 8\%, respectively) (Fig. 2A and 2D). Cur

A)

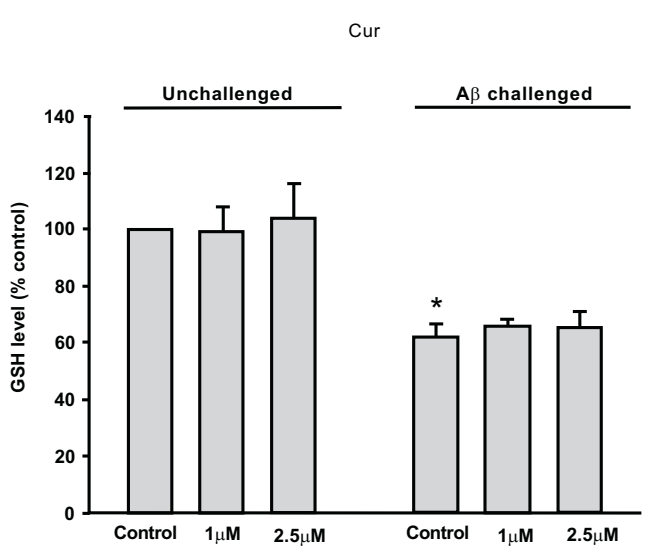

C)

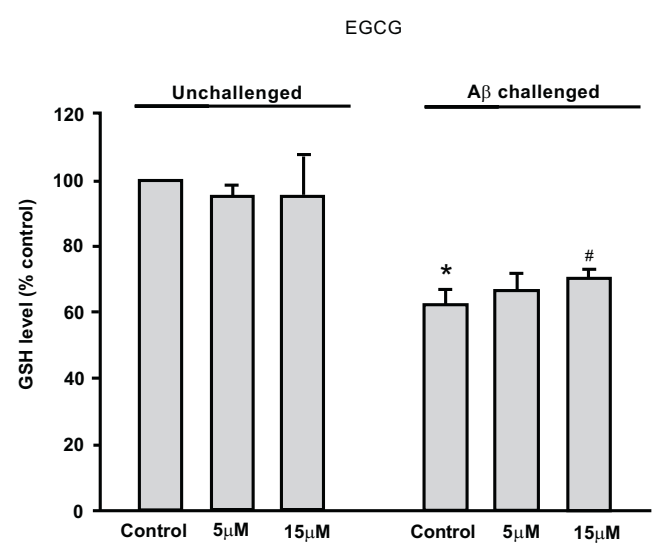

and Rev did not produce any effect on cellular GSH levels in A $\beta$-challenged cells (Fig. 2B and 2C).

$t$-BHP induced a marked GSH depletion (40\%) in the first minute post-challenge in control cells which were not pre-incubated with the phytochemicals. While (-)Sch B and EGCG pre-incubation significantly reduced the degree of GSH depletion (by 72.5 and 25\%, respectively), Cur and Rev pre-incubation did not affect the extent of t-BHP-induced GSH depletion (Fig. 3).

\section{$(-)$ Sch $B$ increases G6PDH and GR activities in A $\beta$ - challenged cells}

Although pre-incubation with phytochemicals did not alter G6DPH and GR activities in unchallenged cells, A $\beta$ challenge significantly decreased G6PDH (37\%) and GR (55\%) activities in SH-SY5Y cells, when compared with unchallenged controls (Fig. 4). Among the tested phytochemicals, only (-)Sch B was able to attenuate the A $\beta$ challenge-induced decreases in G6PDH and GR activities (by 15 and $11 \%$, respectively) (Fig. 4).

\section{B)}

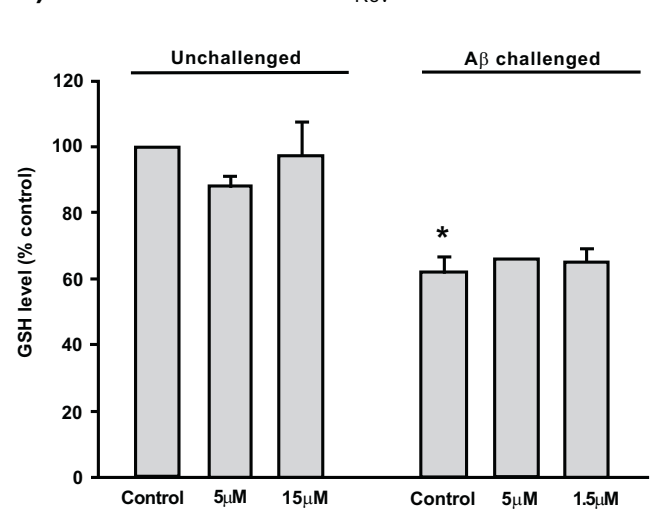

D)

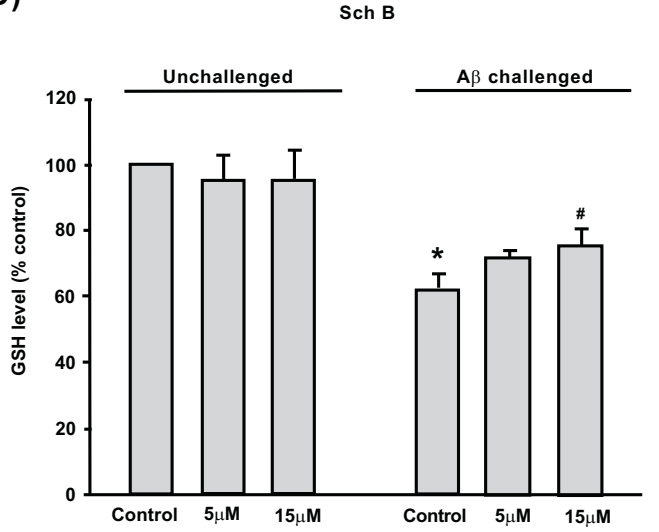

Fig. (2). Effects of (-)Sch B, curcumin, resveratrol and EGCG on cellular GSH levels in A $\beta$-challenged SH-SY5Y cells. Cells were preincubated with different concentrations of phytochemical ( 5 or $15 \mu \mathrm{M}$ for (-)Sch B, resveratrol or EGCG; 1 or $2.5 \mu \mathrm{M}$ for curcumin) prior to $\mathrm{A} \beta$ challenge. Cellular GSH levels were measured as described in methods and materials. Data are expressed as percent control with respect to the unchallenged control value $(18.9 \mathrm{nmol} / \mathrm{mg}$ protein \pm 1.74$)$. Values given are means $\pm \mathrm{SD}$, with $\mathrm{n} \geq 3$. $*$ Significantly different from the unchallenged control group $(P<0.05)$; \# significantly different from the A $\beta$-challenged control group $(P<0.05)$. 

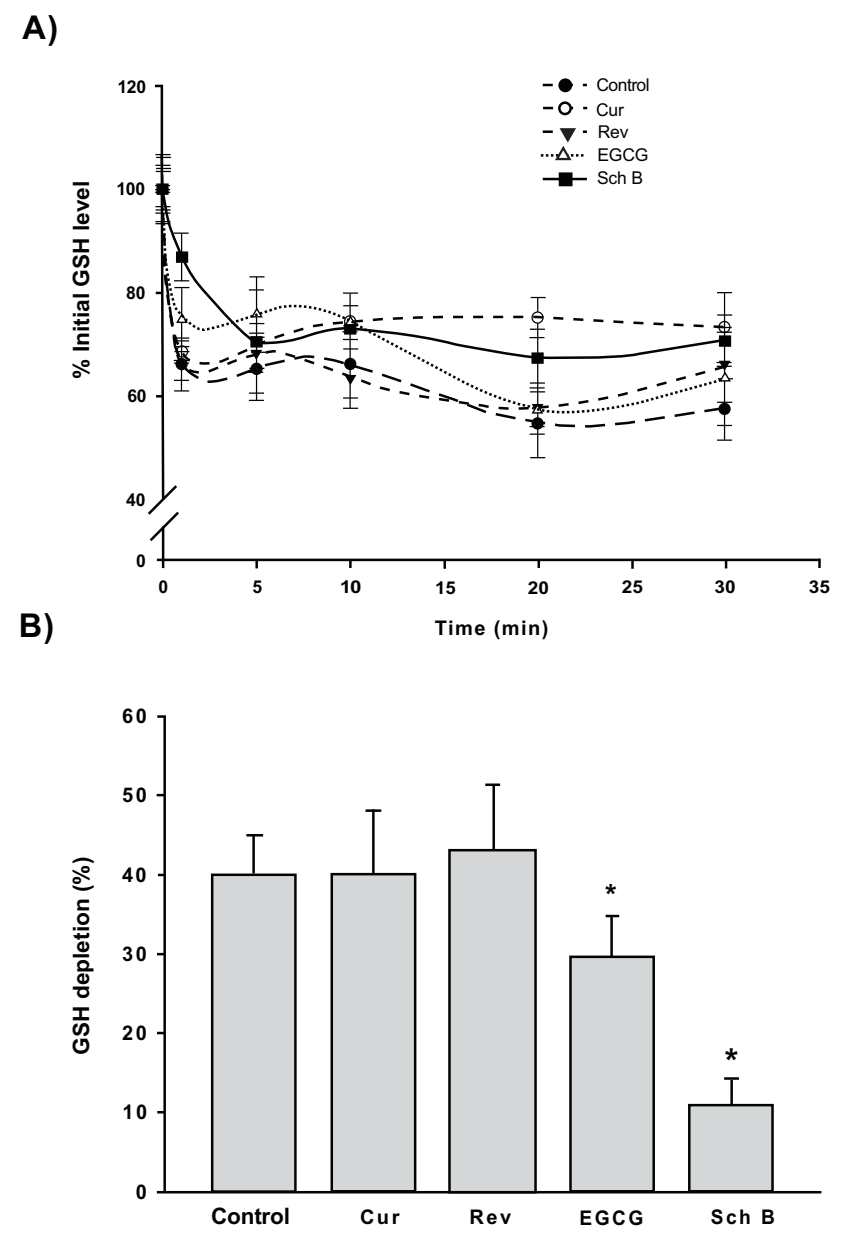

Fig. (3). Effects of (-)Sch B, curcumin, resveratrol, EGCG on peroxide-induced GSH depletion in SH-SY5Y cells. Cells were pre-incubated with different concentrations of phytochemical (15 $\mu \mathrm{M}$ for (-)Sch B, resveratrol or EGCG; $2.5 \mu \mathrm{M}$ for curcumin) prior to $t$-BHP $(1 \mathrm{mM})$ challenge. Control cells were treated with DMSO only. The $t$-BHP-induced depletion of GSH was estimated as described in Materials and methods. Values given are means \pm SD, with $\mathrm{n} \geq 3$. * Significantly different from the control group $(P<0.05)$.

\section{(-)Sch B triggers the activation of $\mathrm{Nrf2}$}

Incubation with (-)Sch B $(15 \mu \mathrm{M})$ or Rev $(15 \mu \mathrm{M})$ led to significant activation of Nrf2 (by 32 and $50 \%$, respectively), whereas Cur $(2.5 \mu \mathrm{M})$ and EGCG $(15 \mu \mathrm{M})$ did not trigger the activation of $\mathrm{Nrf} 2$, as assessed by the luciferase assay (Fig. 5).

\section{(-)Sch B suppresses A $\beta$-induced Tau phosphorylation}

Although pre-incubation with the experimental phytochemicals did not cause any detectable change in the extent of Tau phosphorylation in unchallenged cells, A $\beta$ challenge caused a significant increase (63\%) in Tau phosphorylation, as assessed by Western blot analysis, when compared with unchallenged control cells. (-)Sch B and EGCG reduced the extent of Tau phosphorylation (by 64 and
A)

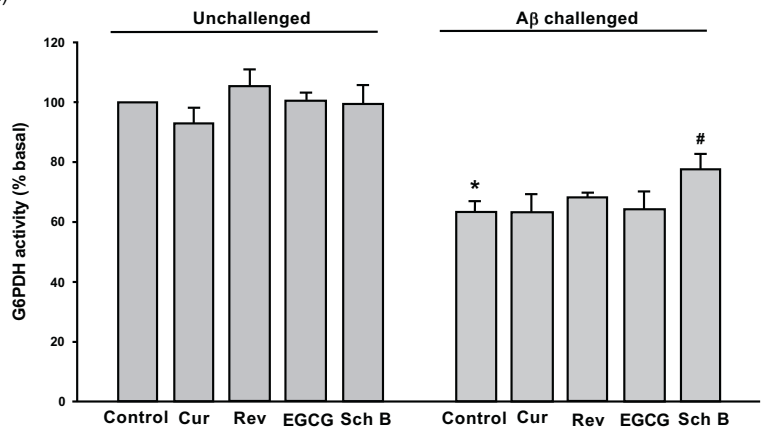

B)

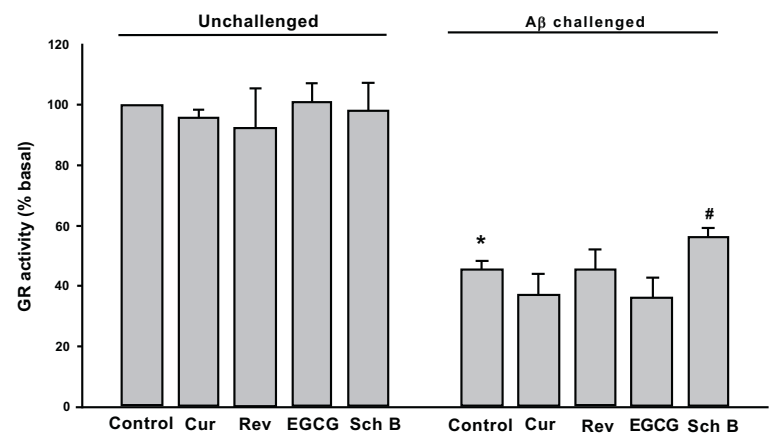

Fig. (4). Effects of (-)Sch B, curcumin, resveratrol and EGCG on G6PDH and GR activities in SH-SY5Y cells. Cells were preincubated with different concentrations of phytochemical $(15 \mu \mathrm{M}$ for (-)Sch B, resveratrol or EGCG; $2.5 \mu \mathrm{M}$ for curcumin) and subjected to $A \beta$ challenge. The activity of (A) glucose-6-phosphate dehydrogenase (G6PDH) and (B) glutathione reductase (GR) were measured as described in Materials and methods. Values given are means $\pm \mathrm{SD}$, with $\mathrm{n} \geq 3$. * Significantly different from the unchallenged control group $(P<0.05)$; \# significantly different from the $\mathrm{A} \beta$ - challenged control group $(P<0.05)$.

68\%, respectively) (Fig. 6). In contrast, Cur and Rev caused only a slight increase in the degree of Tau phosphorylation in A $\beta$-challenged cells (Fig. 6).

\section{DISCUSSION}

Oxidative stress has been recognized as a determining factor in causing neuronal cell death in Alzheimer's disease $[10,21]$. (-)Sch B, a dibenzocyclooctadiene derivative isolated from the fruit of Schisandra chinensis, has been shown to induce an antioxidant response in various organs of rodents and in cultured cell lines, including neuronal cells [5, $16,22]$. In the present study, the ability of (-)Sch B to protect against $\mathrm{A} \beta$-induced apoptosis in human neuroblastoma SH-SY5Y cells was investigated and compared with Cur, Rev, and EGCG, which have also been shown to increase cellular antioxidant capacity. A $\beta$ is a hydrophobic, non-glycosylated peptide containing 39-43 amino acids, which forms a $\beta$-pleated sheet structure [13]. The aggregated insoluble fragments of $A \beta$ constitute the core substance of senile plaques, a hallmark in the post-mortem diagnosis of Alzheimer's disease. In vivo and in vitro studies have shown that $A \beta$ is cytotoxic $[14,23-25]$, so that the 


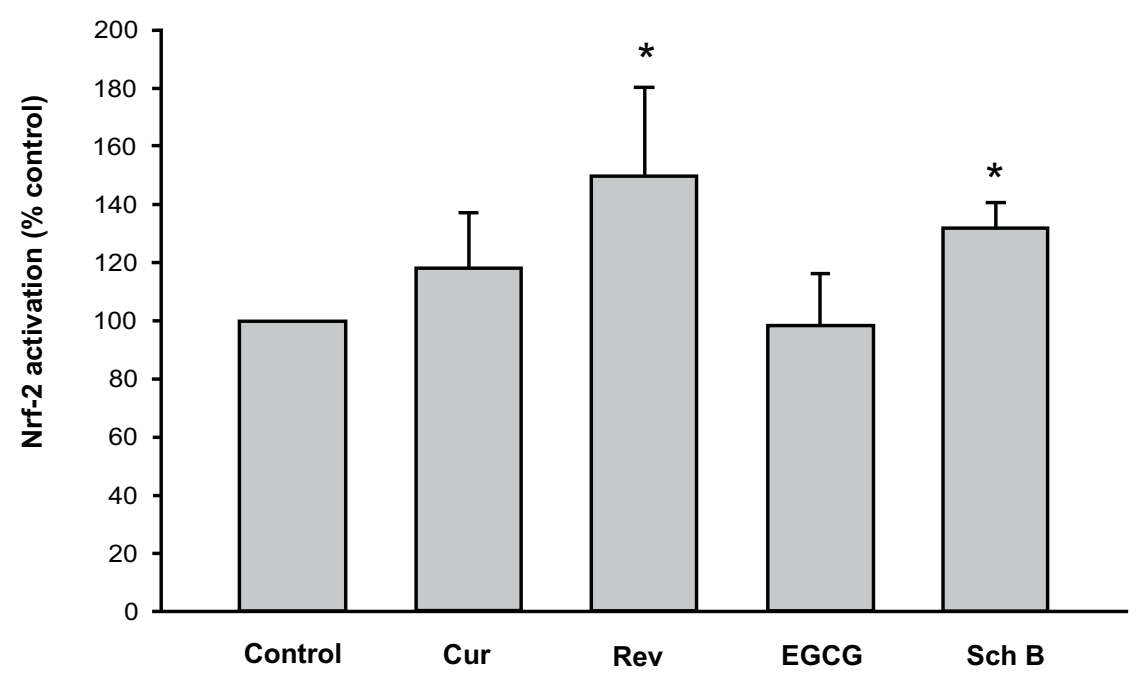

Fig. (5). Effects of (-)Sch B, curcumin, resveratrol and EGCG on Nrf2 activation in SH-SY5Y cells. Cells were pre-incubated with different concentrations of phytochemical $(15 \mu \mathrm{M}$ for $(-)$ Sch B, resveratrol or EGCG; $2.5 \mu \mathrm{M}$ for curcumin), and the activation of Nrf2 was assessed by a luciferase assay as described in Materials and methods. Values given are means $\pm \mathrm{SD}$, with $\mathrm{n} \geq 3$. * Significantly different from the unchallenged control group $(P<0.05)$.

aggregation of $\mathrm{A} \beta$ produces a chronic oxidative stress in neuronal cells and eventually induces apoptotic neuronal cell death [26, 27]. Among the four tested phytochemicals, only $(-)$ Sch B and EGCG were found to protect against $A \beta$ induced apoptosis in SH-SY5Y cells. However, other reports have demonstrated that Cur and Rev can protect against $A \beta$ induced toxicity in primary neurons and neuroblastoma cells [28, 29]. The discrepancy between the present study and earlier studies by other laboratories may be related to differences in drug treatment protocols. In previous studies, exposure to Cur or Rev was immediately followed by A $\beta$ challenge [28, 29], whereas in the present study, phytochemical-pre-incubated cells were further incubated with fresh medium without phytochemicals for $16 \mathrm{~h}$ before being subjected to $A \beta$ challenge. The glutathione antioxidant response, if it occurs, is elicited during the post-drug exposure incubation period. It is also worth noting that Cur produced significant cytotoxicity in $A \beta$-unchallenged cells at the highest concentrations tested (i.e., 5 and $15 \mu \mathrm{M}$ ). Therefore, lower concentrations of Cur (i.e., 1 and $2.5 \mu \mathrm{M}$ ) were used in later experiments. The cytotoxicity produced by Cur in neuroblastoma cells may be related to its ability to induce apoptosis via the inhibition of the nuclear factor kappa B pathway [30].

Since oxidative stress is presumed to play a pivotal role in the pathogenesis of Alzheimer's disease, it is of pharmacological interest to investigate whether or not the cytoprotective actions afforded by (-)Sch B and EGCG are paralleled by an antioxidant response involving the activation of the Nrf2 signaling pathway. Under the experimental conditions used in the present study, (-)Sch B but not EGCG was found to trigger the activation of $\mathrm{Nrf2}$, although cytoprotection was observed in both (-)Sch B and EGCG pre-incubated cells. This observation suggested that different cytoprotective mechanisms may exist for $(-)$ Sch B and EGCG. While (-)Sch B might exert its cytoprotective action by eliciting an antioxidant response involving $\mathrm{Nrf} 2$ activation, the cytoprotection by EGCG against oxidant injury was likely mediated by its free radical scavenging action. However, Rev, which did not protect against $A \beta$ challenge, could also produce activation of $\mathrm{Nrf} 2$ in $\mathrm{SH}-$ SY5Y cells. Conceivably, Rev, which can merely cause the dissociation of Nrf2 and Keap1 [31], did not trigger an antioxidant response leading to the protection of SH-SY5Y cells against $A \beta$ challenge under the present experimental conditions. Clearly, the mechanism underlying the action of Rev remains to be elucidated.

The cytoprotective effects of the tested phytochemicals on apoptosis were assessed by measuring the activation of caspase-3 activity. A study in our laboratory has shown that the caspase-3 activity correlates well with the extent of menadione-induced apoptosis in $\mathrm{H} 9 \mathrm{c} 2$ and AML12 cells (unpublished data). The cytoprotection afforded by (-)Sch B and EGCG was also associated with a decrease in the extent of $\mathrm{A} \beta$ - and $t$-BHP-induced GSH depletion in SH-SY5Y cells, suggesting that $(-)$ Sch B can consistently restore GSH levels under different types of oxidative stress. The reduction in the extent of GSH depletion caused by (-)Sch B was associated with an enhancement in G6PDH and GR activities, indicative of an enhanced glutathione redox cycling. GR is a ubiquitously expressed enzyme which requires NADPH synthesized by G6PDH to reactivate oxidized glutathione (GSSG) by reducing it back to GSH in a timely fashion [32]. In this regard, the ability of GR to regenerate $\mathrm{GSH}$ from GSSG and thereby maintain an appropriate thiol/disulfide homeostasis, particularly during the early exposure to oxidative stress, has been shown to be a crucial factor in determining cell survival under these conditions [33, 34]. Taken together, the present findings further strengthen the notion that Nrf2-regulated glutathione redox cycling is crucial for cell survival [35]. 

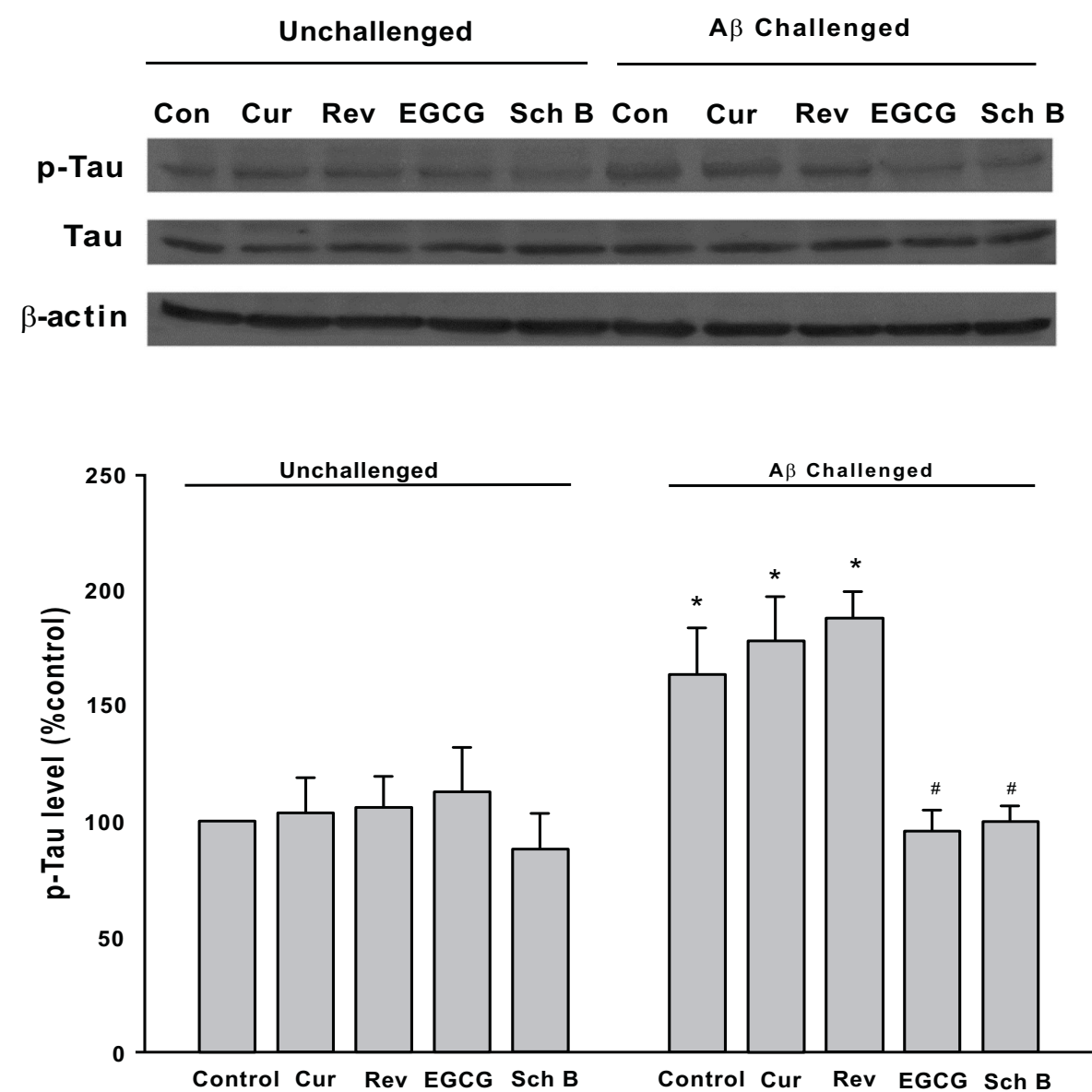

Fig. (6). Effects of (-)Sch B, curcumin, resveratrol and EGCG on Tau phosphorylation in A $\beta$-challenged SH-SY5Y cells. Cells were treated as described in Fig (4). Phosphorylated and total Tau as well as $\beta$-actin (loading control) levels in unchallenged and A $\beta$-challenged cells were examined by Western blot analysis. The quantification of phosphorylated Tau is expressed in $\%$ control with respect to the unchallenged control. Values given are means $\pm \mathrm{SD}$, with $\mathrm{n} \geq 3$. * Significantly different from the unchallenged control group $(P<0.05)$; \# significantly different from the $\mathrm{A} \beta$-challenged control group $(P<0.05)$.

The microtubule-associated protein Tau, which functions to stabilize microtubules, is expressed in brain tissue. Under normal conditions, Tau is minimally phosphorylated in adult brain tissue [36, 37]. However, Tau was found to become hyperphosphorylated in patients with Alzheimer's disease, and the phosphorylated form of Tau has been recognized as a main component of paired helical filaments and thus neurofibrillary tangles (NFTs) $[38,39]$. NFTs are one of the main pathological signatures of Alzheimer's disease and their presence positively correlates with the severity of dementia in this condition [40]. Studies from various laboratories have shown that oxidative stress results in the hyperphosphorylation of Tau in both animal and cultured neuronal cell models [41-43]. It is noteworthy in this regard that the present study has indicated that both $(-)$ Sch B and EGCG can suppress the hyperphosphorylation of Tau through the reduction of cellular oxidative stress levels, as indicated by a reduction in GSH depletion under $A \beta$ challenged conditions. Whereas (-)Sch B may enhance cellular glutathione redox cycling, as evidenced by increases in G6PDH and GR activities, the antioxidant effect of EGCG is likely due to its free radical scavenging activity. Taken together, the ability of (-)Sch B and EGCG to reduce the extent of Tau phosphorylation under conditions of $A \beta$ challenge suggests their potential beneficial effect in ameliorating the pathological symptoms of Alzheimer's disease.

\section{CONCLUSION}

Despite the fact that a range of phytochemicals can produce an antioxidant response in cells, the mechanism of action characteristic of a given phytochemical limits its ability to protect against different types of oxidative stress and hence limits its application. Among the four phytochemicals tested, only (-)Sch B and EGCG were able to protect against $\mathrm{A} \beta$ challenge-induced apoptosis in $\mathrm{SH}$ SY5Y cells. While the (-)Sch-B afforded cytoprotection is likely mediated by Nrf2-regulated enhancement of cellular glutathione redox cycling, EGCG may exert its cytoprotective action by virtue of its direct free radical scavenging properties.

\section{CONFLICT OF INTEREST}

The authors confirm that this article content has no conflict of interest. 


\section{ACKNOWLEDGEMENTS}

Declared none.

\section{REFERENCES}

[1] Nordberg A. Pharmacological treatment of cognitive dysfunction in dementia disorders. Acta Neurol Scand Suppl 1996; 168: 87-92.

[2] McDowell I. Alzheimer's disease: insights from epidemiology. Aging (Milano) 2001; 13: 143-62.

[3] Christen Y. Oxidative stress and Alzheimer disease. Am J Clin Nutr 2000; 71: 621-9S.

[4] Kim J, Lee HJ, Lee KW. Naturally occurring phytochemicals for the prevention of Alzheimer's disease. J Neurochem 2010; 112: 1415-30.

[5] Peng Y, Xing C, Lemere CA, et al. 1-3-n-Butylphthalide ameliorates beta-amyloid-induced neuronal toxicity in cultured neuronal cells. Neurosci Lett 2008; 434: 224-9.

[6] Wobser H, Dussmann H, Kogel D, et al. Dominant-negative suppression of HNF-1 alpha results in mitochondrial dysfunction, INS-1 cell apoptosis, and increased sensitivity to ceramide-, but not to high glucose-induced cell death. J Biol Chem 2002; 277: 641321.

[7] Chiu PY, Leung HY, Poon MKT, Ko KM. Chronic schisandrin B treatment improves mitochondrial antioxidant status and tissue heat shock protein production in various tissues of young adult and middle-aged rats. Biogerontology 2006; 7: 199-210.

[8] Chiu PY, Luk KF, Leung HY, et al. Schisandrin B stereoisomers protect against hypoxia/reoxygenation-induced apoptosis and inhibit associated changes in $\mathrm{Ca} 2+$-induced mitochondrial permeability transition and mitochondrial membrane potential in H9c2 cardiomyocytes. Life Sci 2008; 82: 1092-101.

[9] Lam PY, Ko KM. (-)Schisandrin B ameliorates paraquat-induced oxidative stress by suppressing glutathione depletion and enhancing glutathione recovery in differentiated PC12 cells. Biofactors 2011; 37: 51-7.

[10] Lam PY, Ko KM. Beneficial effect of (-)schisandrin B against 3nitropropionic acid-induced cell death in PC12 cells. Biofactors 2012; 38: 219-25.

[11] Kelsey NA, Wilkins HM, Linseman DA. Nutraceutical antioxidants as novel neuroprotective agents. Molecules 2010; 15: 7792-814.

[12] Butterfield DA. Amyloid beta-peptide (1-42)-induced oxidative stress and neurotoxicity: implications for neurodegeneration in Alzheimer's disease brain. A review. Free Radic Res 2002; 36: 1307-13.

[13] Lin MT, Beal MF. Alzheimer's APP mangles mitochondria. Nat Med 2006; 12: 1241-43.

[14] Loo DT, Copani A, Pike CJ, et al. Apoptosis is induced by betaamyloid in cultured central nervous system neurons. Proc Natl Acad Sci USA 1993; 90: 7951-5.

[15] Alía M, Ramos S, Mateos R, Bravo L, et al. Response of the antioxidant defense system to tert-butyl hydroperoxide and hydrogen peroxide in a humanhepatoma cell line (HepG2). J Biochem Mol Toxicol 2005; 19: 119-28.

[16] Lam PY, Leong PK, Chen N, Ko KM. Schisandrin B enhances the glutathione redox cycling and protects against oxidant injury in different types of cultured cells. Biofactors 2011; 37: 439-46.

[17] Chiu PY, Leung HY, Poon MKT, et al. (-)Schisandrin B is more potent than its enantiomer in enhancing cellular glutathione and heat shock protein production as well as protecting against oxidant injury in H9c2 cardiomyocytes. Mol Cell Biochem 2006; 289: 18591.

[18] Griffith OW. Biologic and pharmacologic regulation of mammalian glutathione synthesis. Free Radic Biol Med 1999; 27: 922-35.

[19] Seelig GF, Meister A. Glutathione biosynthesis: gammaglutamylcysteine synthetase from rat kidney. Methods Enzymol 1985; 113: 379-90.

[20] Ip SP, Poon MKT, Wu SS, et al. Effect of schisandrin B on hepatic glutathione antioxidant system in mice: protection against carbon tetrachloride toxicity. Planta Med 1995; 61: 398-401.

[21] Sultana R, Perluigi M, Butterfield DA. Protein oxidation and lipid peroxidation in brain of subjects with Alzheimer's disease: insights into mechanism of neurodegeneration from redox proteomics. Antioxid Redox Signal 2006; 8: 2021-37.

Ko KM, Lam BY. Schisandrin B protects against tertbutylhydroperoxide induced cerebral toxicity by enhancing glutathione antioxidant status in mouse brain. Mol Cell Biochem 2002; 238: 181-6.

[23] Forloni G, Chiesa R, Smiroldo S, et al. Apoptosis mediated neurotoxicity induced by chronic application of beta amyloid fragment 25-35. Neuroreport 1993; 4: 523-6.

[24] Giordano T, Pan JB, Monteggia LM, et al. Similarities between beta amyloid peptides 1-40 and 40-1: effects on aggregation, toxicity in vitro, and injection in young and aged rats. Exp Neurol 1994; 125: 175-82.

[25] Kowall NW, McKee AC, Yankner BA, Beal MF. In vivo neurotoxicity of beta-amyloid [beta(1-40)] and the beta(25-35) fragment. Neurobiol Aging 1992; 13: 537-42.

[26] Zhu X, Raina AK, Lee HG, et al. Oxidative stress signalling in Alzheimer's disease. Brain Res 2004; 1000: 32-9.

[27] Zhu X, Raina AK, Perry G, Smith MA. Alzheimer's disease: the two-hit hypothesis. Lancet Neurol 2004; 3: 219-26.

[28] Han YS, Zheng WH, Bastianetto S, et al. Neuroprotective effects of resveratrol against beta-amyloid-induced neurotoxicity in rat hippocampal neurons: involvement of protein kinase $\mathrm{C} . \mathrm{Br} \mathrm{J}$ Pharmacol 2004; 141: 997-1005.

[29] Huang HC, Xu K, Jiang ZF. Curcumin-mediated neuroprotection against amyloid-beta-induced mitochondrial dysfunction involves the inhibition of GSK-3beta. J Alzheimers Dis 2012; 32: 981-96.

[30] Freudlsperger C, Greten J, Schumacher U. Curcumin induces apoptosis in human neuroblastoma cells via inhibition of NFkappaB. Anticancer Res 2008; 28: 209-14.

[31] Taguchi K, Motohashi H, Yamamoto M. Molecular mechanisms of the Keap1-Nrf2 pathway in stress response and cancer evolution. Genes Cells 2011; 16: 123-40.

[32] Meister A. Glutathione metabolism and its selective modification. J Biol Chem 1988; 263: 17205-8.

[33] Smith AC, Boyd MR. Preferential effects of 1,3-bis(2-chloroethyl)1-nitrosourea (BCNU) on pulmonary glutathione reductase and glutathione/glutathione disulfide ratios: possible implications for lung toxicity. J Pharmacol Exp Ther 1984; 229: 658-63.

[34] Doroshenko N, Doroshenko P. Ion dependence of cytotoxicity of carmustine against PC12 cells. Eur J Pharmacol 2003; 476: 185-91.

[35] Harvey CJ, Thimmulappa RK, Singh A, et al. Nrf2-regulated glutathione recycling independent of biosynthesis is critical for cell survival during oxidative stress. Free Radic Biol Med 2009; 46: 443-53.

[36] Goedert M, Jakes R, Crowther RA, et al. Epitope mapping of monoclonal antibodies to the paired helical filaments of Alzheimer's disease: identification of phosphorylation sites in tau protein. Biochem J 1994; 301: 871-7.

[37] Goedert M, Wischik CM, Crowther RA, et al. Cloning and sequencing of the cDNA encoding a core protein of the paired helical filament of Alzheimer disease: identification as the microtubule-associated protein tau. Proc Natl Acad Sci USA 1988; 85: 4051-5.

[38] Crowther RA, Olesen OF, Smith MJ, et al. Assembly of Alzheimer-like filaments from full-length tau protein. FEBS Lett 1994; 337: 135-8.

[39] Mandelkow EM, Mandelkow E. Tau in Alzheime's disease. Trends Cell Biol 1998; 8: 425-7.

[40] Arriagada PV, Growdon JH, Hedley-Whyte ET, Hyman BT. Neurofibrillary tangles but not senile plaques parallel duration and severity of Alzheimer's disease. Neurology 1992; 42: 631-9.

[41] Lovell MA, Xiong $\mathrm{S}$, Xie $\mathrm{C}$, et al. Induction of hyperphosphorylated tau in primary rat cortical neuron cultures mediated by oxidative stress and glycogen synthase kinase-3. J Alzheimers Dis 2004; 6: 659-71.

[42] Olivieri G, Brack C, Muller-Spahn F, et al. Stahelin HB. Mercury induces cell cytotoxicity and oxidative stress and increases betaamyloid secretion and tau phosphorylation in SHSY5Y neuroblastoma cells. J Neurochem 2000; 74: 231-6. 
[43] Melov S, Adlard PA, Morten K, et al. Johnson F. Mitochondrial oxidative stress causes hyperphosphorylation of tau. PLoS One 2007; 2: e536.

Received: August 29, 2014

Revised: October 23, 2014

Accepted: October 30, 2014

(C) Leong et al.; Licensee Bentham Open.

This is an open access article licensed under the terms of the Creative Commons Attribution Non-Commercial License (http://creativecommons.org/licenses/by-nc/3.0/) which permits unrestricted, non-commercial use, distribution and reproduction in any medium, provided the work is properly cited. 